\title{
Update Schmerzrechtsprechung: Stellungnahme aus ärztlicher Sicht
}

\section{Jörg Jeger}

Dr. med., Facharzt für Rheumatologie, FMH, EMBA, MAS Versicherungsmedizin, Chefarzt MEDAS Zentralschweiz

Ab den 1980er Jahren ist es zu einem massiven Anstieg vorzeitiger Berentungen gekommen. Leider haben sich die medizinischen Fachgesellschaften dieser sozialpolitisch wichtigen Frage zu lange nicht angenommen, so dass das Bundesgericht 2004 eingriff und den Riegel schob. Es bewahrheitete sich eine alte Weisheit der Swissair aus den 1950er Jahren: «Wer nicht fliegt, wird überflogen.»

\section{Die Beurteilung der Arbeitsfähigkeit: eine vernachlässigte Aufgabe}

Die Beurteilung der Arbeitsfähigkeit wird offensichtlich in der ärztlichen Ausbildung immer noch sträflich vernachlässigt. Dies zeigte eine kürzlich in dieser Zeitung veröffentlichte Umfrage unter Facharztabsolventinnen und -absolventen [1]. Unter 23 erfragten Ausbildungszielen erhielt die Beurteilung der Arbeitsfähigkeit in allen Fachbereichen die schlechtesten Bewertungen. Hier besteht dringender Nachholbedarf in der Aus- und Weiterbildung.

\section{Nachteile der bisherigen Schmerzrecht- sprechung («Überwindbarkeitspraxis»)}

- Die empirische Datenbasis für die bundesgerichtliche Vermutung, somatoforme Störungen seien "willentlich überwindbar», ist sehr dünn, der Verweis auf die "medizinische Empirie» nicht haltbar. [2]

- Problematisch ist der Zusammenzug der erwähnten Krankheitsbilder unter dem Begriff "pathogenetisch-ätiologisch unklare syndromale Beschwerdebilder ohne nachweisbare organische Grundlage». Es handelt sich dabei um eine juristische Begriffsbildung, die in der Medizin so nicht gebräuchlich ist.

- Das Bundesgericht hat die Kriterien für eine schlechte Prognose, die vom Tübinger Psychiater Klaus Foerster stammten, erheblich abgeändert und anders gewichtet. So erhielt die psychiatrische Komorbidität einen Stellenwert, der den medizinischen Originalen nicht entnommen werden kann. Sie wurde zum eigentlichen "Killerkriterium» eines Rentenantrages.
- Diese Rechtsprechung führte in aller Regel zu Allesoder-nichts-Entscheiden. Die medizinische Situation von Exploranden mit psychosomatischen Leiden ist aber oft so, dass sie noch über Ressourcen verfügen, die sie für ein Erwerbsleben sinnvoll nutzen könnten. Diese reichen aber nicht aus, um ein Vollzeitpensum in der freien Wirtschaft zu bestehen.

- Mangelnde Objektivierbarkeit korreliert nicht per se mit «willentlicher Überwindbarkeit».

- Die alte Schmerzrechtsprechung wurde für bestimmte Diagnosen konzipiert. Diesem Vorgehen liegt die Fehlannahme zu Grunde, dass eine Diagnose Rückschlüsse zulässt auf das Ausmass der Behinderung.

- Die alte Überwindbarkeitspraxis war den Versicherten gegenüber schwer zu kommunizieren und hat zu beleidigenden und entwürdigenden Formulierungen geführt. Sie hat auch viel zur Verhärtung der Fronten zwischen den Organen der Invalidenversicherung und den praktizierenden Ärztinnen und Ärzten beigetragen, was in dieser Zeitung mehrmals thematisiert wurde.

Wendepunkt: Das Urteil 9C_492/2014 vom 3. Juni 2015

Das Wichtigste in Kürze:

- Die Überwindbarkeitsvermutung wird, da für sie keine genügende wissenschaftliche Grundlage besteht, aufgegeben.

- Das Bundesgericht spricht neu von «anhaltender somatoformer Schmerzstörung und vergleichbaren psychosomatischen Leiden". 
- Das frühere Regel-Ausnahme-Verfahren wird ersetzt durch ein strukturiertes Beweisverfahren anhand vorgegebener Indikatoren. Die psychiatrische Komorbidität wird in ihrer Bedeutung stark relativiert, der primäre Krankheitsgewinn ganz aufgegeben.

- Diese Elemente werden bewusst nicht Kriterien, sondern Indikatoren genannt. Jeder Fall, unabhängig von der Diagnose, muss ergebnisoffen gleich seriös geprüft werden.

- Anhand der Indikatoren müssen behandelnde und begutachtende Ärztinnen und Ärzte darlegen, inwieweit funktionelle Einschränkungen mit Auswirkungen auf Aktivität und Partizipation bestehen. Die Indikatoren betreffen den funktionellen Schweregrad der Erkrankung und die Konsistenz der Behinderung (gleichmässige Einschränkungen des Aktivitätsniveaus in vergleichbaren Lebensbereichen, Leidensdruck).

- Die neue Rechtsprechung legt Wert darauf, dass nicht nur die Defizite (negatives Leistungsbild), sondern auch die Ressourcen (positives Leistungsbild) erfasst und dargelegt werden. Diese neue Sichtweise gipfelt im Satz: "Arbeitsunfähigkeit leitet sich aus dem Saldo aller wesentlichen Belastungen und Ressourcen $a b . "$

- Die antragstellende Person bleibt beweispflichtig: Wenn der konsistente Beweis einer Behinderung nicht erbracht werden kann, so sind keine Versicherungsleistungen (insbesondere Renten) geschuldet.

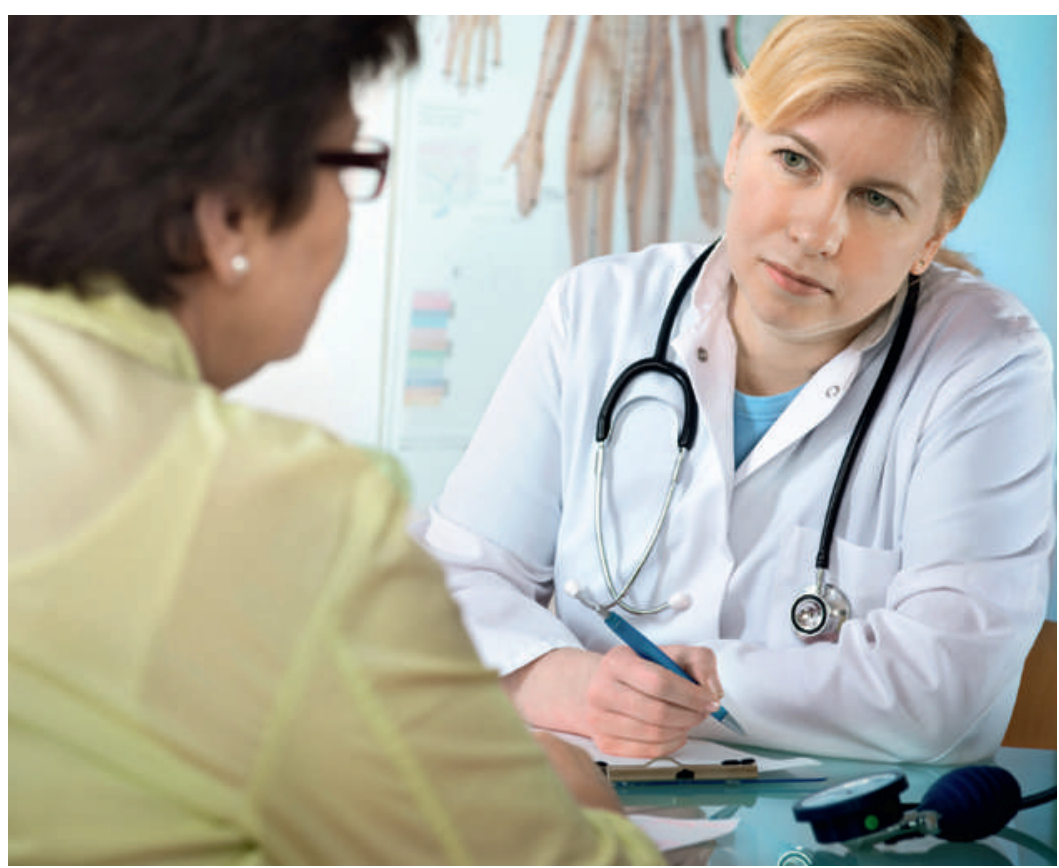

Die neue Rechtsprechung legt Wert darauf, dass nicht nur die Defizite, sondern auch die Ressourcen erfasst und dargelegt werden.

\section{Eine erste Würdigung aus ärztlicher Sicht}

Das Bundesgericht hat früher als erwartet und ziemlich radikal zu Fehlern Stellung genommen und diese ausgeräumt. Mit der neuen Rechtsprechung zu psychosomatischen Krankheitsbildern ist ihm ein wegweisender, moderner "grosser Wurf» gelungen. Praktisch sämtliche in den vergangenen 10 Jahren aus medizinischer und juristischer Optik vorgebrachten Mängel wurden auf einen Schlag behoben. Dabei ist das Bundesgericht mit erstaunlicher Offenheit und Selbstkritik vorgegangen.

\section{Das Bundesgericht hat früher als erwartet und ziemlich radikal zu Fehlern Stellung genommen und diese ausgeräumt.}

- Man findet darin das Eingeständnis, die alte Rechtsprechung habe dazu verleitet, den Regelfall als gegeben anzunehmen und zu verwirklichen. In diesem Zusammenhang spricht das Bundesgericht gar von einem «Bias» (systematischen Fehler), was aufhorchen lässt.

- Das strukturierte Beweisverfahren anhand von Indikatoren ist ergebnisoffen. Beurteilende Ärzte wie auch der Rechtsanwender haben somit mit gleicher Sorgfalt, unabhängig von der Diagnose, zu prüfen, ob eine Behinderung vorliegt oder nicht. Dass dabei erforderlich ist, dass diese Behinderung konsistent und in vergleichbaren Lebensbereichen analog zu bestehen hat, muss auch aus ärztlicher Sicht betont und unterstützt werden.

- Der Rechtsanwender fordert weiterhin eine objektive Zumutbarkeitsbeurteilung. Gemeint ist wohl, dass nicht allein das betroffene Subjekt die Leistungseinschränkung begründen kann, sondern dass die verschiedenen Fachleute aus dem medizinischen Bereich und der beruflichen Eingliederung zum Schluss kommen müssen, dass eine reale für den Berufsalltag relevante Behinderung vorliegt. Damit bekommen Beobachtungen aus professionell begleiteten Eingliederungsversuchen einen höheren Stellenwert.

- Die wohl grösste und in meinen Augen schönste Änderung besteht im Menschenbild, das der neuen Rechtsprechung zugrunde liegt: Alle am Verfahren Beteiligten müssen der antragstellenden Person mit der gleichen Sorgfalt und demselben Respekt begegnen, unabhängig von den Diagnosen. Es gibt keine "Geht-uns-sowieso-nichts-an-Diagnosen" (GUSNAD) mehr und eine leistungsablehnende Verfügung oder ein Gerichtsurteil sollte auch keine persönlichkeitsverletzenden Formulierungen mehr enthalten. 
- Die neue Rechtsprechung verlegt den Hauptfokus von der Diagnose auf den Nachweis der Behinderung (funktionelle Einschränkungen mit Auswirkungen auf Aktivität und Partizipation).

- Das Urteil ist geprägt von einem modernen Medizin- und Rehabilitationsverständnis: Behinderung entsteht aus der Wechselwirkung zwischen dem von Krankheit oder Unfallfolgen betroffenen Individuum und seiner Umwelt. Ob ein Mensch seine gesellschaftlichen Verpflichtungen wahrnehmen kann oder nicht, hängt vom Verhältnis zwischen den funktionellen Einschränkungen (Defiziten), den persönlichen Ressourcen und den Anforderungen des Lebens ab.

\section{Das Bundesgericht erwartet von den Fach- gesellschaften Leitlinien zur Beurteilung psychosomatischer Störungen.}

- Die neue Rechtsprechung stellt hohe Anforderungen sowohl an die beurteilenden Ärztinnen und Ärzte wie auch an die Rechtsanwender. Bleibt zu hoffen, dass alle Beteiligten diesen Anforderungen genügen können. Die Ärzteschaft wird die Ausbildung im Hinblick auf die Beurteilung der Arbeitsfähigkeit und die Implementierung der ICF vertiefen müssen. Dabei sind besonders die Fachgesellschaften und die FMH gefordert. Das Bundesgericht hat auch unüberhörbar dargelegt, dass es von den Fachgesellschaften Leitlinien zur Beurteilung psychosomatischer Störungen erwartet. Je mehr sich die Rechtsprechung auf einen tragfähigen Konsens der Fachverbände stützen kann, desto weniger sind eigene juristische Hilfskonstruktionen nötig. Das sollten sich die Ärzte hinter die Ohren schreiben!
- Gewisse Unklarheiten bestehen insofern, als erst die Zukunft zeigen wird, wo der Rechtsanwender die Schwelle für den erbrachten Beweis der Behinderung anlegt. Hier besteht ein Ermessensspielraum, der wohl von verschiedenen Seiten ausgelotet wird.

- Die neue Rechtsprechung darf auf keinen Fall als Türöffner für eine grosszügige Berentung analog zu den 1990er Jahren missverstanden werden. Es kann nicht genügend betont werden: Gefordert ist der konsistente Nachweis der Behinderung, nicht mehr, aber auch nicht weniger. Die Ärzteschaft sollte aus der Vergangenheit die nötigen Lehren ziehen. Tut sie dies nicht, wird sie bestimmt ein nächstes Mal (und dann vermutlich definitiv) übergangen. Mit der neu zurückgewonnenen Verantwortung müssen wir mit aller Sorgfalt und Umsicht umgehen!

- Die Juristen Thomas Gächter und Michael Meier haben in einem jüngst veröffentlichten Jusletter [3] von "Zeichen einer hohen Justizkultur» gesprochen. Ich kann ihnen dabei uneingeschränkt beipflichten. Als Mediziner wie auch als Staatsbürger möchte ich diese Anerkennung mit einem grossen Dank verbinden. Es ist ein modernes Urteil, das den Fortschritten der Medizin und Rehabilitation in weiten Teilen Rechnung trägt.

\section{Literatur}

1 Siegrist M. et al. Beurteilung der Weiterbildung durch Fachärztinnen und Fachärzte. Schweiz Ärztezeitung. 2015;96(22):758-63.

2 Jeger J. Die Entwicklung der «FOERSTER-Kriterien» und ihre Übernahme in die bundesgerichtliche Rechtsprechung: Geschichte einer Evidenz. Jusletter vom 16.5.2011. www.jusletter.ch

3 Gächter T, Meier ME. Schmerzrechtsprechung 2.0. Jusletter vom 29.06.2015. www.jusletter.ch

Bildnachweis

Alexander Raths | Dreamstime.com 